\title{
TORQUE CHARACTERIZATION TEST AND SHOCK ANALYSIS OF HINGE MECHANISM DESIGNED FOR SMALL SATELLITES
}

\author{
Srinath.M.K ${ }^{1}$ \\ ${ }^{I}$ Research Scholar, Assistant professor, Dept of mechanical engineering, NHCE, Bangalore
}

\begin{abstract}
Dynamic tests and shock analysis tests are the basic tests required for any mechanisms. The tests were carried out to study the hinge mechanism which was designed for small satellites. Such mechanisms can be used for the deployment of the solar panels, reflectors, antennas etc. The present hinge mechanism was designed for the deployment of solar panels. Dynamic test characterization of the hinge mechanism for different condition showed that there was almost no loss of deployable torque. The shock test gave a clear view of the latching characteristics. Also the deployment time was measured accurately which is not possible with standard methods since the value is very small in the order of a few mille-seconds. The deployment shock characteristic of this particular hinge mechanism could be clearly noticed with the shock test results which showed a drop in $G$ force during latching of the flat spring.
\end{abstract}

\section{INTRODUCTION}

A new hinge mechanism was required towards the development of small satellite and to test the mechanism for torque characterization and shock analysis. In this direction a number of satellite mechanisms were studied from different journals and papers [1-6]. The mechanisms examined included were the hold and release mechanism, which consisted of hold down bolt and plunger. When the plunger is removed from the slot in the hold down bolt, it releases the panels. Another mechanism studied was the closed control loop which consisted of pulleys and wire, which on straightening would open up the panels. Another special type of mechanism is the eddy current damper which develops a magnetic force which opposes the driving torque. Upon observing and studying some of these mechanisms, a hinge mechanism was designed and developed for small satellites, where these mechanisms could be fitted into small spaces. The present paper deals with the torque characterization test and shock analysis of the hinge mechanism.

During the torque characterization test the aiding and resisting torque present in the hinge was measured. The torsion spring provides the aiding torque and frictional torque in the hinge is the resisting torque. The hinge torque characteristics were studied for different conditions. The hinge mechanism was analyzed for four different configurations. The configurations were four different combinations of the flat spring (FS) and Torsional spring (TS), i.e., with or without flat spring and torsion spring. The torque was obtained by calculating the product between the force (obtained experimentally using a force gauge) and the perpendicular distance (obtained by measuring the effective distance between the axis of the shaft and the point at which the value of force is picked up). Using different values of torque for stow and deploy condition, both the nominal torque (drive torque) and frictional torque (resistive torque) were calculated. Fig 1.1 shows the hinge mechanism, with the flat spring. Fig 1.2 shows the hinge mechanism, with torsional spring.

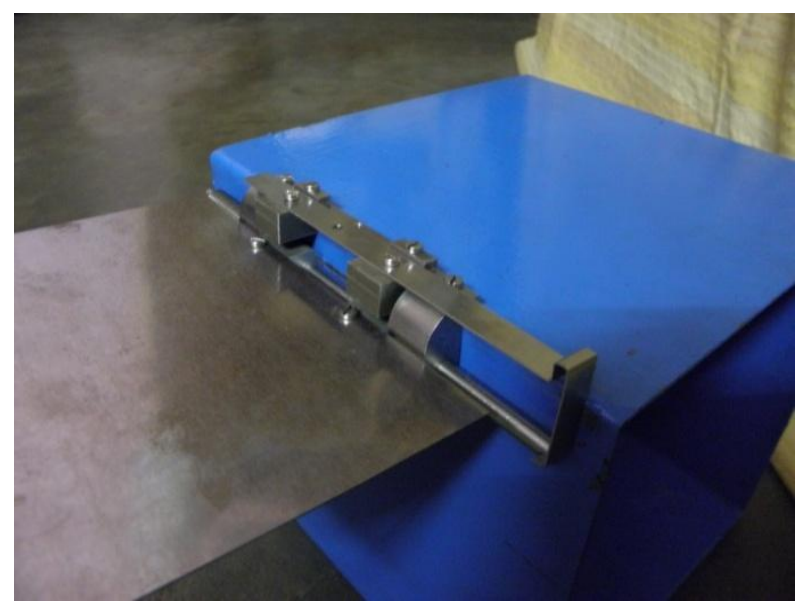

Fig 1.1: Hinge mechanism (Flat spring)

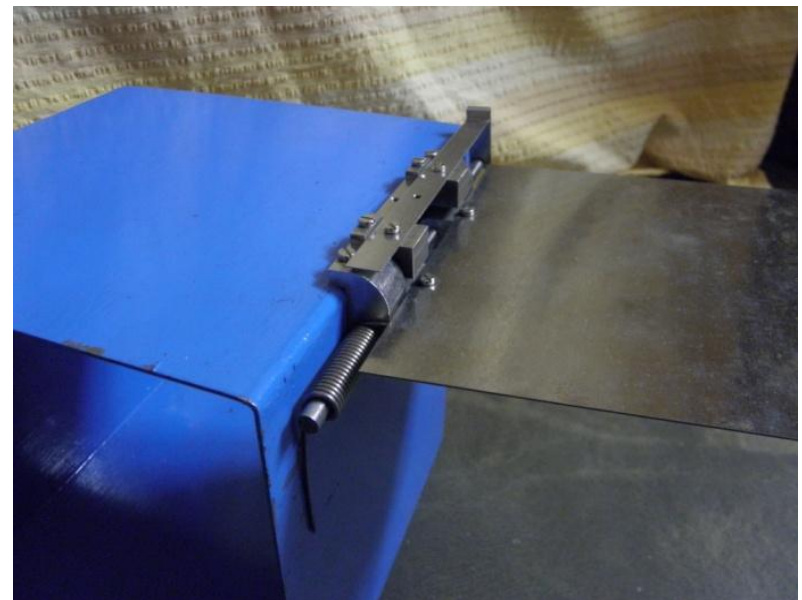

Fig 1.2: hinge mechanism (Torsional spring) 
Shock testing replicates events to determine if structures can withstand sudden applied forces. They are characterized by their short duration and sudden occurrence. Shock is usually measured by an accelerometer. This describes a shock pulse as a plot of acceleration versus time. Acceleration can be reported in units of metre per second squared. Often, for convenience, the magnitude of a shock is stated as a multiple of the standard acceleration due to free fall in the Earth's gravity, a quantity with the symbol ' $g$ ' having the value $9.80665 \mathrm{~m} / \mathrm{s}^{2}$. Thus a shock of " $20 \mathrm{~g}$ " is equivalent to about $196 \mathrm{~m} / \mathrm{s}^{2}$. A shock can be characterized by the peak acceleration, the duration, and the shape of the shock pulse. The design of the hinge mechanism was carried out taking care that the shock on the panel be minimized. Shock analysis was carried out to understand and study the behavior of the system to sudden shock in different points on the panels and the mechanism.

\section{EXPERIMENTAL SET UP FOR DYNAMIC}

\section{TESTING}

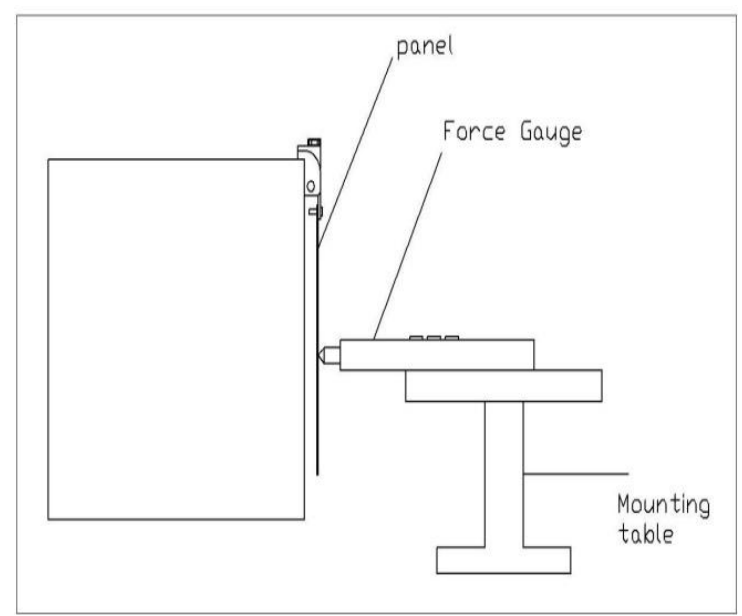

Fig 2.1: Experimental set up for dynamic testing

The fundamentals were studied carefully from a journal presented by Zhao yang et al [7]. For the present research, the experimental set up consisted of an electronic force gauge mounted on a movable fixture board, that slided on a table along one axis. The amount of movement of the movable board was precisely controlled with the help of a micrometer attached to it. Hence the movement of the force gauge along one axis could be controlled up to a minimum of $0.01 \mathrm{~mm}$. The prototype was locked in position with the help of fixtures on a table. Data was obtained by a single point of the force gauge that was positioned to act on the panel. With the help of the micrometer, the force gauge acted in both directions to get the required data. Fig2.1 shows the experimental set up for the measurement of the forces.

Since we know that Torque $=$ Force $\mathrm{x}$ Perpendicular distance. Hence, the torque was obtained by calculating the product between the force (obtained experimentally using a force gauge) and the perpendicular distance (obtained by measuring the effective distance between the axis of the shaft and the point at which the value of force is picked up).
Using different values of torque for stow and deploy condition, both the nominal torque (drive torque) and frictional torque (resistive torque) were calculated.

\section{RESULTS AND DISCUSSIONS ON DYNAMIC TESTING}

The hinge mechanism was analyzed for four different configurations. Tests were carried out for Flats Springs of $0.5 \mathrm{~mm}$ thickness, material SS and a free length of $65 \mathrm{~mm}$. The graphs show the results measured under various assembly conditions of flat spring and the torsional spring.

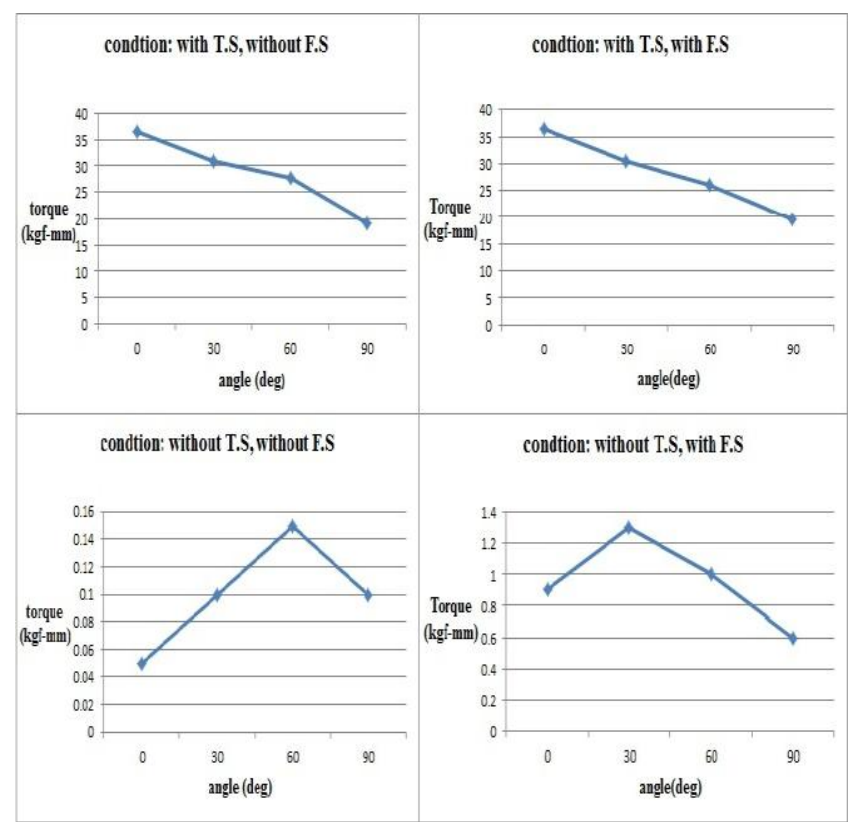

Fig 3.1: Graph of Torque v/s time

We can see that the torque drops as the angle of deployment increases for condition 1 and 2. Condition 3 and 4 behaves differently with an increase in torque up to angle of deployment 60degrees and 30 degrees respectively, beyond which there is a drop. Since the torsion spring is absent, the friction between the bearing surfaces dominate deployment in condition 3 . However in condition 3 the y-axis scale value is low i.e. friction torque is very low throughout the deployment. Also the spring torque available is high enough to overcome the frictional torque and ensure positive deployment. While in condition 4 the absence of torsion spring and presence of Flat spring results in more friction being produced. In condition 2 since the flat spring is present the deployment is more uniform with respect to the other conditions.

\section{EXPERIMENTAL SET UP FOR SHOCK}

\section{ANALYSIS}

The requisites for the understanding of shock testing was examined by the papers presented by Andrew Higgins et al [8] and Christian Laa [9]. The experimental setup consisted of four accelerometers positioned on four pre-defined points on the prototype for the present investigation. Two placed on the panels and two on the hinge mechanism gave 
different shock values corresponding to its position. All four accelerometers were connected to a power amplifier for data amplification. The amplified data was passed on to the data acquisition system connected to the display system (computer) which in turn plotted different values of ' $g$ ' against time. Besides giving the acceleration values, the graphs also identify accurate deployment time. Care was taken while wiring the accelerometers to make sure that wire movement (during deployment) does not influence the readings. This was done by taping the wire to minimize movement.

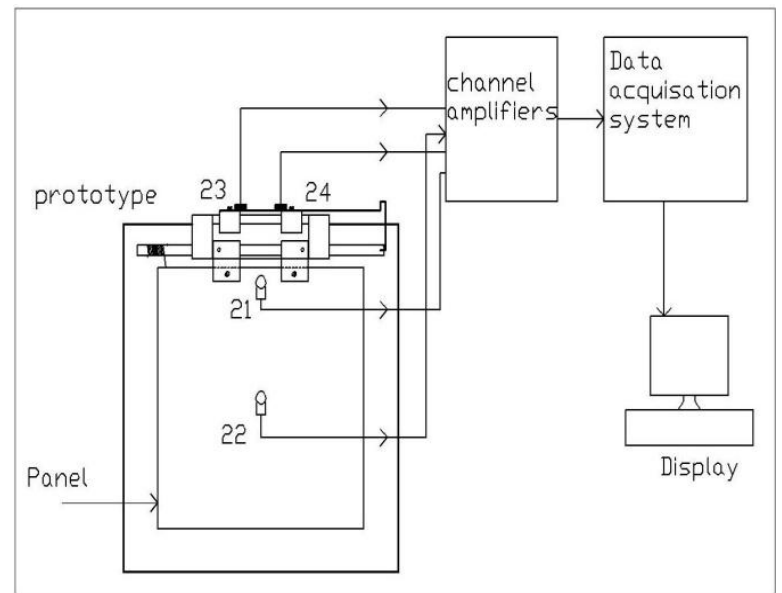

Fig 4.1: Experimental set up for shock analysis.

\section{RESULTS AND DISCUSSIONS ON SHOCK}

\section{ANALYSIS}

The shock experienced by the system was analyzed by studying the graphs which consisted of responses obtained by plotting different values of ' $G$ ' force against time' $t$ '.

$$
\mathrm{G}=\mathrm{A} / \mathrm{g}
$$

Where $\mathrm{G}=$ Constant, $\mathrm{A}=$ Resultant acceleration $\left(\mathrm{m} / \mathrm{s}^{2}\right), \mathrm{g}=$ acceleration due to gravity $\left(\mathrm{m} / \mathrm{s}^{2}\right)$
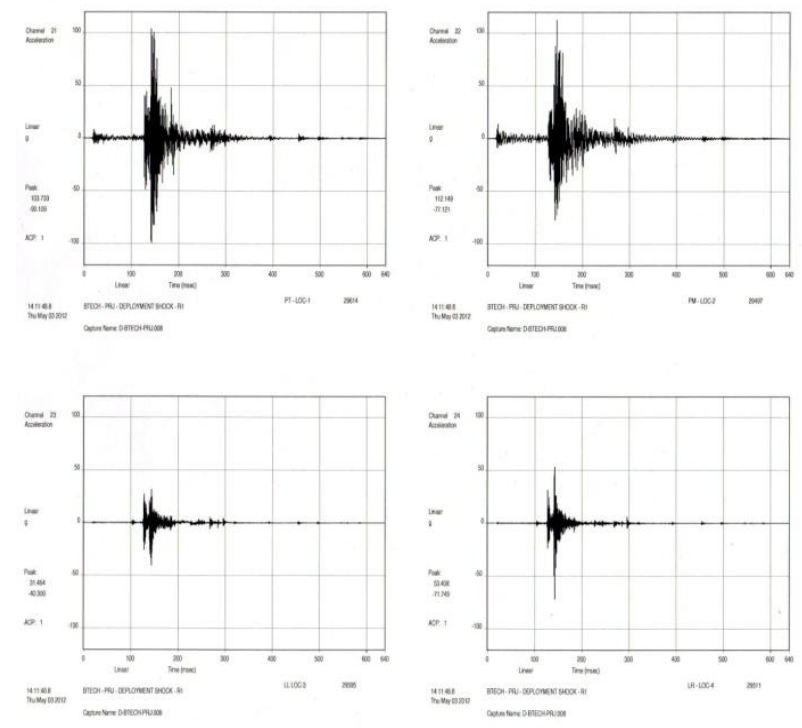

Fig 5.1: Results of shock analysis with Flat spring
From the graph of Fig 5.1 and Fig 5.2, it is understood that during time, $\mathrm{t}=0$ millisecs to 120 milli-seconds, the panel is deploying. Hence 120 milli seconds is the deployment time. At about 120 milli-seconds, latching occurs during which that panel experiences a peak value of 103.733 (top) and 112.149 (mid) Gs. It is clear from the other two graphs that the flat spring absorbs some energy on latching, since it experiences peak value of 31.464 (left) and 53.408 (right) Gs .
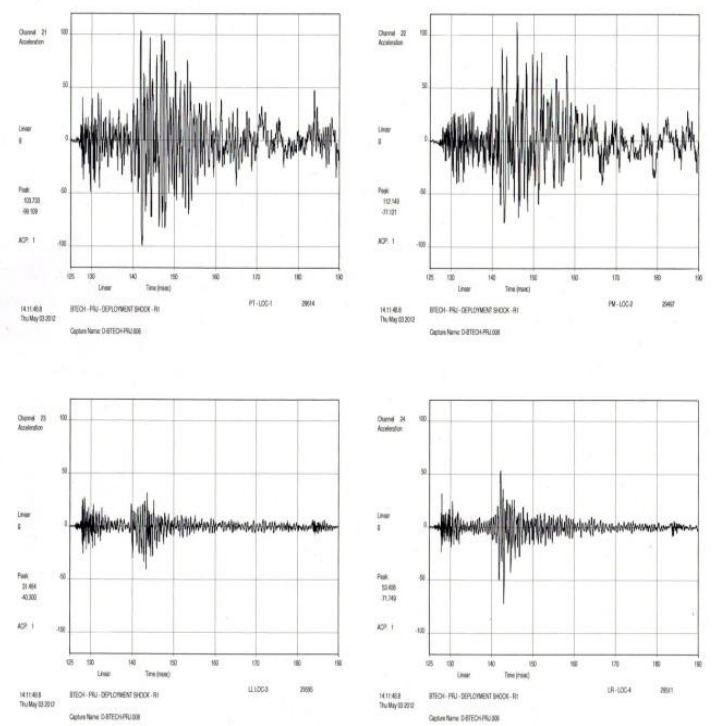

Fig 5.2: Peak loads of shock analysis with Flat spring

\section{CONCLUSION}

Characterization of the hinge mechanism for different condition showed that there was almost no loss of deployable torque for different conditions. It also gave a clear view of how the system behaved with change in configurations. Also, the torque that the flat spring experienced during latching could be calculated accurately which helped in designing the same more efficiently.

The shock test gave a clear view of the latching characteristics. Also the deployment time was measured accurately which is not possible with standard methods since the value is very small in the order of a few milliseconds. One very important factor in designing a hinge mechanism was to minimize deployment shock (on components). This characteristic of this particular hinge mechanism could be clearly noticed with the shock test results which showed a drop in $\mathrm{G}$ force during latching of the flat spring.

\section{SCOPE FOR FUTURE WORK}

The materials used for the development of this hinge mechanism were solely chosen for ground testing purpose. They do not fulfill the space environment conditions. The model was created to understand the concept of this particular Hinge mechanism and analyze the system under different conditions. With this, the system can be characterized and the reliability and functioning can be 
understood in detail. As the system is considered to function efficiently and is reliable, then the material designing for space purpose can be carried out. The hinge mechanism could be used for actual space applications.

\section{REFERENCES}

[1] Developmeny of a reusable cubesat satellite bus architecture for KYSAT-1 spacecraft. Tyler James Doering University of Kentucky, 2009, Pages 1-5

[2] Solar Array Deployment Mechanism Mark C. Calassa - and Russell Kackley" Lockheed Missiles and Space Company, Inc., Sunnyvale, CA, 1990, Pages 80-85

[3] Design, Construction and Validation of Articulated solar panels of CubeSat, Patrick Hohn, Lulea University of Technology, 2010, Pages 40-45

[4] Analysis for the Design of Satellites in LEO, TanjaNemetzade, Engineering Systems Division, Massachusetts Institute of Technology,2020, pages 110

[5] Development of the Aquarius Antenna Deployment Mechanisms, Joel A Johnson, Jet Propulsion Laboratory, California Institute of Technology, Pasadena, CA, 2008, pages 23-34

[6] No-shock hold-down and release mechanism, Christian Laa, Theodor Nitschko, Ludwig SupperGerhard Traxler, Jean-Michel Lautier, European Space Mechanisms \& Tribology Symposium - ESMATS 2011, pages 385-397

[7] Dynamic testing method for space docking mechanism. Zhao Yang, Cao Xi-Bin, Sun Zhao-Wei Aircraft Engineering and aerospace technology, Vol 75, issue 6, pg 600-604

[8] A Simple Shock Wave-Based Mechanism for Forward-Accelerated fragment generation from AntiSatellite Impacts. By Andrew Higgins, Oren Petel, Francois-Xavier Jette. Shock Wave Physics Group.

[9] UNLIMITED RESETTABLE NO-SHOCK HOLDDOWN AND RELEASE MECHANISM. By Christian Laa, Theodor Nitschko, Ludwig Supper, Gerhard Traxler, Jean-Michel Lautier. European space mechanism and symposium.2011. 